King's College KCL-TH-96-17

hepth@xxx/9611075

August 8, 2018

\title{
Superconformal Invariants and Extended Supersymmetry
}

\author{
P.S. Howe \\ and \\ P.C. West \\ Department of Mathematics \\ King's College, London
}

\begin{abstract}
The superconformal invariants in analytic superspace are found. Superconformal invariance is shown to imply that the Green's functions of analytic operators are invariant holomorphic sections of a line bundle on a product of certain harmonic superspaces. It is argued that the correlation functions for a class of sufficiently low dimension gauge invariant operators in $N=2$ and $N=4$ supersymmetric Yang-Mills theory can be evaluated up to constants.
\end{abstract}


It is well-known that supersymmetric Yang-Mills theories with $N=4$ supersymmetry possess remarkable properties. Several arguments have been advanced which suggest that these theories are finite in perturbation theory [1] and even non-perturbatively. They are therefore quantum-mechanically superconformally invariant. Moreover [6], $N=4$ theories have the right structure to exhibit Olive-Montonen duality [9].

In a recent paper [3], we argued that one might be able to exploit the symmetry properties of these theories further to calculate the Green's functions for certain gauge-invariant operators and we produced evidence which indicated that these might be rather simple. In this note we give further details on these results. We show explicitly how to compute the Green's functions, and how the constraints imposed by the analyticity properties of the theory severely restrict the possible functions that can arise. It turns out that there are no arbitrary functions of superconformal invariants that can occur in certain Green's functions and hence that they are determined up to constants. Our computations are at the conformal point, i.e. there is no symmetry breaking, but our results nevertheless suggest that the theory away from the conformal point might also be rather simple. In addition the results are restricted to a certain sector in the theory, the so-called analytic sector, but we emphasize that this sector is much larger than the chiral sectors of $N=1$ or $N=2$ gauge theories. In particular, the operators we consider include the energy-momentum tensor multiplet, as well as all other gauge-invariant polynomials of the analytic field strength multiplet.

We begin with a brief review of the theory which we will then reformulate in two different, but related, ways. The basic multiplet consists of six scalar fields, four left-handed chiral fermions and the gauge field, all of which fields transform under the adjoint representation of the gauge group which we can take to be $S U(M)$ to be definite. If we replace the gauge field by its field strength tensor, the entire multiplet can be assembled into an $N=4$ superfield $W_{i j}, i, j=1 \ldots 4$, which is antisymmetric on its $S U(4)$ indices and which obeys the following constraints:

$$
\begin{aligned}
\nabla_{\alpha i} W_{j k} & =\nabla_{\alpha[i} W_{j k]} \\
\nabla_{\dot{\alpha}}^{i} W_{j k} & =-\frac{2}{3} \delta_{[j}^{i} \nabla_{\dot{\alpha}}^{l} W_{k] l}
\end{aligned}
$$

as well as the reality condition

$$
\bar{W}^{i j}=\frac{1}{2} \epsilon^{i j k l} W_{k l}
$$

where the bar includes hermitian conjugation for the Lie algebra basis matrices. The latter equation implies that only one of the first two is independent. These equations involve the superspace gauge-covariant derivative $\nabla_{\alpha i}$, and hence are quite complicated to write out explicitly. Nevertheless, when one defines the components of $W_{i j}$ as the fields obtained by successively evaluating spinorial covariant derivatives of $W_{i j}$ at $\theta=0$ one finds exactly the components listed above.

A more useful formulation of $N=4$ Yang-Mills theory uses harmonic superspace [4]. It was observed in [5] that one could rewrite the above constraints in a simpler form if one uses an appropriate harmonic superspace. If we let $M$ denote $N=4$ superspace, the harmonic superspace we wish to consider is $M_{H}=M \times \mathbb{F}$ where $\mathbb{F}$ is an internal space 
which we take to be the Grassmannian of two-planes in $\mathbb{C}^{4}, \mathbb{G}_{2}(4)$. It can be realised as a homogeneous space of $S U(4)$ by $\mathbb{F}=H \backslash S U(4)$ where $H=S(U(2) \times U(2))$. Following Gikos [4], we sometimes find it convenient to work on the space $\hat{M}_{H}:=M \times S U(4)$ with the understanding that all fields will be equivariant with respect to the left action of the isotropy subgroup, that is, $F(h u)=M(h) F(u)$, where $u \in S U(4), h \in H$, and where $M$ denotes a represention of $H$ acting on the representation space $V$, say, of $H$ in which $F$ takes its values. A set of "coordinates" for $\hat{M}_{H}$ is given by $x^{\alpha \dot{\alpha}}, \theta^{\alpha i}, \bar{\theta}_{i}^{\dot{\alpha}}$ and $u_{I}^{i}$, where $u \in S U(4)$ and where the capital index is acted on by the isotropy group. We write $u_{I}^{i}=\left(u_{r}^{i}, u_{r^{\prime}}{ }^{i}\right)$ where $r=1,2$ and $r^{\prime}=3,4$. The inverse of $u$ is denoted $u_{i}^{I}=\left(u_{i}^{r}, u_{i}{ }^{r^{\prime}}\right)$. The right invariant vector fields on $S U(4)$ are denoted $\tilde{D}_{I}^{J}$, where $\tilde{D}_{I}^{I}=0$. They generate the left action of $S U(4)$ on itself, from which fact one can deduce the following simple formulae for their action on the $u$ 's:

$$
\begin{aligned}
& \tilde{D}_{I}^{J} u_{K}^{k}=\delta_{K}^{J} u_{I}^{k}-\frac{1}{4} \delta_{I}^{J} u_{K}^{k} \\
& \tilde{D}_{I}^{J} u_{k}^{K}=-\delta_{I}^{K} u_{k}^{J}+\frac{1}{4} \delta_{I}^{J} u_{k}^{K}
\end{aligned}
$$

For applications to the coset space we are interested in, we separate the derivatives into a set corresponding to the isotropy group, $\left\{D_{r}^{s}, D_{r^{\prime}} s^{\prime}, D_{o}\right\}$, and a set corresponding to the coset space, $\left\{D_{r}^{s^{\prime}}, D_{r^{\prime}}{ }^{s}\right\}$. The notation here is that $D_{r}{ }^{s}$ and $D_{r^{\prime}} s^{\prime}$ are both traceless (i.e. correspond to $\mathfrak{s u}(2)$ 's) and the $\mathfrak{u}(1)$ derivative $D_{o}$ is normalised so that

$$
D_{o} u_{r}^{i}=\frac{1}{2} u_{r}^{i} \quad D_{o}{u_{r^{\prime}}}^{i}=-\frac{1}{2} u_{r^{\prime}}{ }^{i}
$$

The space $\mathbb{F}$ is a complex manifold with complex dimension 4 ; the derivatives $D_{r^{\prime}}{ }^{s}$ are essentially equivalent to the holomorphic derivatives on $\mathbb{F}$ while the derivatives $D_{r}{ }^{s^{\prime}}$ are their complex conjugates and correspond to the antiholomorphic derivatives on $\mathbb{F}$.

Returning to Yang-Mills theory, we define the superfield $W$ by

$$
W:=\epsilon^{r s} u_{r}^{i} u_{s}^{j} W_{i j}
$$

It has the following properties: firstly, it is covariantly Grassmann-analytic, (G-analytic), which means that

$$
\nabla_{\alpha r} W=\nabla_{\dot{\alpha}}^{r^{\prime}} W=0
$$

where small internal indices are converted to large ones and hence to $r, r^{\prime}$ by means of $u_{I}^{i}$ and its inverse; secondly, it is analytic in its dependence on the coordinates of $\mathbb{F}$, (F-analytic),

$$
D_{r}^{s^{\prime}} W=0
$$

thirdly, it has $U(1)$ charge 1 , so that $D_{o} W=W$, and finally it is real with respect to a certain real structure on $M_{H}$. The latter is defined as follows: for any function $F(u)$ on $S U(4)$, we set $\widetilde{F}(u):=\overline{F(k u)}$, where $k$ is the matrix

$$
\left(\begin{array}{rr}
0 & 1 \\
-1 & 0
\end{array}\right)
$$


written in block two-by-two form. For $W$, the reality constraint $W=\widetilde{W}$ is equivalent to the self-duality constraint in ordinary superspace. We also remark that the converse holds, namely, given a field $W$ satisfying (6), (7) and $D_{o} W=W, W=\widetilde{W}$, it can be written in the form (5) for a $W_{i j}$ satisfying (1) and (2).

The idea is to consider gauge-invariant operators of the form $A_{m}:=\operatorname{tr}\left(W^{m}\right), m=2 \ldots M$. These have the property that they are ordinarily $G$-analytic, i.e.

$$
D_{\alpha r} A_{m}=D_{\dot{\alpha}}^{r^{\prime}} A_{m}=0
$$

as well as being analytic on $\mathbb{F}$ and real. Clearly $A_{m}$ has $U(1)$ charge $m$. The most interesting operator in this set is the first one $A_{2}:=T$, the energy-momentum tensor multiplet. It has $128+128$ components including the conserved currents for all the symmetries of the superconformal group.

In order to calculate the Green's functions for these operators it is convenient to reformulate the theory once more. This is most easily accomplished by working in complex spacetime, which is not a drawback since one can easily re-impose reality or one can take the view that these functions should anyway be defined in certain regions of products of complex spacetimes according to the general axioms of quantum field theory. The complex superspace relevant for us can be constructed as a coset space of the complexified superconformal group $S L(4 \mid 4, \mathbb{C})$. This is not a simple group but it is easier to work with than its simple cousin because it can be written in terms of matrices. One can check that for the cosets of interest only the simple part acts non-trivially. The space we are interested in is the super-Grassmannian $\mathbb{G}_{2 \mid 2}(4 \mid 4)$, i.e. the space of $(2 \mid 2)$-planes in $N=4$ supertwistor space $\mathbb{T}_{4} \cong \mathbb{C}^{4 \mid 4}$. The analytic functions on this space correspond precisely to analytic continuations of the type of functions we have introduced above, i.e. the $G$-analytic and $\mathbb{F}$-analytic functions on harmonic superspace. This superspace can be presented as a coset space of $S L(4 \mid 4)$ [5] [3] with isotropy group $P$ consisting of supermatrices of the form

$$
\left(\begin{array}{cccc|cccc}
\times & \times & & & \times & \times & & \\
\times & \times & & & \times & \times & & \\
\times & \times & \times & \times & \times & \times & \times & \times \\
\times & \times & \times & \times & \times & \times & \times & \times \\
\hline \times & \times & & & \times & \times & & \\
\times & \times & & & \times & \times & & \\
\times & \times & \times & \times & \times & \times & \times & \times \\
\times & \times & \times & \times & \times & \times & \times & \times
\end{array}\right)
$$

where the crosses denote elements which are not necessarily zero. One can think of the coset itself as corresponding to the blank portions of the above diagram. Notice that both the spacetime and internal parts of the body, corresponding to the top left and bottom right blocks respectively, have the same form, namely the coset space $P_{o} \backslash S L(4)$ where $P_{o}$ 
is the subgroup of $S L(4)$ consisting of matrices of the form

$$
\left(\begin{array}{cccc}
\times & \times & & \\
\times & \times & & \\
\times & \times & \times & \times \\
\times & \times & \times & \times
\end{array}\right)
$$

This is just the space $\mathbb{F}=\mathbb{G}_{2}(4)$. Observe that the number of odd dimensions of the above superspace (corresponding to the off-diagonal blocks) is 8 , half of that of complex super Minkowski space. Therefore one can think of fields on this space as being fields defined on complex super Minkowski space times the internal space $\mathbb{F}$, and satisfying the Grassmann analyticity condition (), or at least the complex version of it.

A slightly different description of the superspace $\mathbb{G}_{(2 \mid 2)}(4 \mid 4)$ is as follows: let $e_{\underline{A}}$ denote a fixed set of basis elements for $\mathbb{T}_{4}$, where $\underline{A}$ is a superindex taking (4|4) possible values. Any (2|2)-plane is specified by a set of basis elements $f_{A}$ where $A$ is a superindex taking $(2 \mid 2)$ values. One can then write

$$
f_{A}=u_{A} \underline{A} e_{\underline{A}}
$$

thus obtaining a description of the plane by the $(2 \mid 2) \times(4 \mid 4)$ supermatrix $u$, which is required to have super-rank $(2 \mid 2)$. The group $S L(4 \mid 4)$ f acting to the left on $\mathbb{T}_{4}$ sends a given plane into another one, and this corresponds to a right action on the matrix $u$. However, such a transformation might simply mix the basis elements of a given plane, so that we can describe the space of $(2 \mid 2)$ planes as the space of matrices of the above type, which we shall denote by $\mathbb{U}$, modulo the left action of $G L(2 \mid 2)$. By exploiting this fact one can show that the space can be covered by coordinate patches of the form

$$
\mathbb{C}^{8 \mid 8} \ni X \mapsto s(X):=\left(\begin{array}{ll}
1 & X
\end{array}\right)
$$

where 1 denotes the $(2 \mid 2) \times(2 \mid 2)$ unit matrix and $X$ the $(2 \mid 2) \times(2 \mid 2)$ matrix of coordinates:

$$
X=\left(\begin{array}{ll}
x & \lambda \\
\pi & y
\end{array}\right)
$$

Here, each entry is a two-by-two matrix, $x$ is the usual spacetime coordinate in twocomponent spinor form, $y$ a local coordinate for the internal space $\mathbb{F}\left(=\mathbb{G}_{2}(4)\right)$ and $\lambda$ and $\pi$ are the fermionic coordinates, which correspond to the $G$-analytic projections of $\theta$ and $\bar{\theta}$ respectively. An important point here is that we wish to consider a subspace of the superGrassmannian which will be non-compact as far as the spacetime part is concerned, but compact as far as the internal part is concerned, i.e. the body will be complex spacetime $\times \mathbb{F}$. It is well-known that complex spacetime can itself be represented as a subset of $\mathbb{G}_{2}(4)$, but not the whole of it unless one wishes to consider its compactified form. To summarise, we shall consider a subspace $\mathbb{M}$ of the super-Grassmanian which can be covered by coordinate patches of the above form such that the body is the required one.

\footnotetext{
${ }^{1}$ We suppress the $\mathbb{C}$ for all groups from now on.

${ }^{2}$ Since this action is transitive, one can recover the coset space description by finding the isotropy group $P$ which is simply the one given above.
} 
We shall not give the details of this here as it will suffice, for our present purposes at least, to consider only one such patch.

The theory is particularly simple in this formulation. The action of the superconformal group on the coordinates $X$ is easily obtained, by sending $s(X)$ to $s(X) g$, where $g \in$ $S L(4 \mid 4)$ and then making a compensating transformation with an element $h(X, g) \in$ $G L(2 \mid 2)$ to restore the form of the function $s$. This gives

$$
s\left(X^{\prime}\right)=h(X, g) s(X) g
$$

where $X^{\prime}$ is the transformed coordinate matrix. Infinitesimally, with $g \simeq 1+\delta g, h \simeq 1+\delta h$, where $\delta g \in \mathfrak{s l}(4 \mid 4)$ and $\delta h \in \mathfrak{g l}(2 \mid 2)$, and with $X^{\prime} \simeq X+\delta X$, one finds

$$
\delta X=B+X D+A X+X C X
$$

and

$$
\delta h=-(A+X C)
$$

where

$$
\delta g=\left(\begin{array}{ll}
-A & B \\
-C & D
\end{array}\right)
$$

A field $f$ on $\mathbb{M}$ is equivalent to an equivariant field $F$ on $\mathbb{U}$, that is a field $F(u)$ such that $F(h u)=M(h) F(u)$ where $M(h)$ now denotes a representation of $G L(2 \mid 2)$ acting on the vector space in which $F$ takes its values. The (local) correspondence is

$$
f(X)=F(s(X))
$$

The left action of the superconformal group on $F$ is given by $F(u) \mapsto F^{\prime}(u)=F(u g)$, and this induces the following action on $f$ :

$$
f(X) \mapsto f^{\prime}(X):=M(h(X, g)) f\left(X^{\prime}\right)
$$

from which one can easily work out the infinitesimal version explicitly. We shall only be concerned with fields which transform under one-dimensional representations of $H$, i.e. fields $F$ such that

$$
F(h u)=(\operatorname{sdet} h)^{-q} F(u)
$$

where $q \in \mathbb{Z}$ is the $U(1)$ charge of the field in the real version given above. For such fields one has the infinitesimal transformation in coordinate form,

$$
\delta f=V f+q \Delta f
$$

where

$$
V=\delta X \frac{\partial}{\partial X}
$$

and $\Delta=-\operatorname{str} \delta h$.

Given this formalism we can state the Ward identities for superconformal invariance for fields of the above type succinctly. A Green's functions of $n$ fields with charges $q_{i}, i=1 \ldots n$ corresponds to a function $F$ on $\mathbb{U}^{n}$ which satisfies the following equation:

$$
F\left(h_{1} u_{1}, \ldots, h_{n} u_{n}\right)=\prod_{i=1}^{n}\left(\operatorname{sdet} h_{i}\right)^{-q_{i}} F\left(u_{1} g, \ldots, u_{n} g\right)
$$


for all $h_{i} \in G L(2 \mid 2)$ and $g \in S L(4 \mid 4)$.

The problem is therefore to solve this equation. We shall do this by first finding a set of quasi-invariants, i.e. functions on $\mathbb{U}^{n}$ invariant under $S L(4 \mid 4)$ and under $S L(2 \mid 2)^{n}$. Consider first $S L(4 \mid 4)$ invariance. The $n$ u's can be considered as a set of vectors in $\mathbb{T}$ (of both even and odd type); if we select out two points, 1 and 2, say, we can form the square matrix $u_{12}$ which we assume to be non-singular:

$$
u_{12}:=\left(\begin{array}{l}
u_{1} \\
u_{2}
\end{array}\right)
$$

One can then show that the differential equation expressing the invariance of a function of these variables under the action of the super Lie algebra $\mathfrak{s l}(4 \mid 4)$ is solved by the following quantities:

$$
\begin{aligned}
s_{12} & :=\operatorname{sdet} u_{12} \\
K_{i} & :=u_{i}\left(u_{12}^{-1}\right)^{1}, i=3 \ldots n \\
L_{i} & :=u_{i}\left(u_{12}^{-1}\right)^{2}, i=3 \ldots n
\end{aligned}
$$

where the 1 and 2 superscripts on the inverse matrix denote its projections onto the corresponding subspaces. Any $\mathfrak{s l}(4 \mid 4)$ invariant will then be a given as a function of these, at least when $u_{12}$ is non-singular. The superdeterminant $s_{12}$ is invariant under $S L_{i}(2 \mid 2)$ for all $i=1 \ldots n$, (where $S L_{i}(2 \mid 2)$ denotes the $S L$ group associated with point $i$ ), and so is already a quasi-invariant. The other quantities transform as

$$
\begin{aligned}
K_{i} & \mapsto h_{i} K_{i} h_{1}^{-1} \\
L_{i} & \mapsto h_{i} L_{i} h_{2}^{-1}
\end{aligned}
$$

We now consider invariance under $S L_{i}(2 \mid 2)$, for each $i=3, \ldots n$. We can apply the same procedure for each $i$ successively to obtain the following sets of variables:

$$
\begin{aligned}
k_{i} & :=\operatorname{sdet} K_{i}, i=3, \ldots n \\
M_{i} & :=\left(K_{i}\right)^{-1} L_{i}, i=3, \ldots n
\end{aligned}
$$

assuming the $K_{i}$ to be invertible. The superdeterminants here are again quasi-invariants while the $M_{i}$ transform as

$$
M_{i} \mapsto h_{1} M_{i} h_{2}^{-1}
$$

We can now solve for $S L_{2}(2 \mid 2)$ invariance by singling out $M_{3}$, say, to get

$$
\begin{aligned}
m_{3} & :=\operatorname{sdet} M_{3} \\
N_{i} & :=M_{i} M_{3}^{-1}, i=4, \ldots n
\end{aligned}
$$

Again, $m_{3}$ is a quasi-invariant while $N_{i}$ transforms as

$$
N_{i} \mapsto h_{1} N_{i} h_{1}^{-1}
$$

Finally we need to find the invariants of the $N_{i}$ under the above action of $S L_{1}(2 \mid 2)$. Clearly the supertrace of $N_{i}$ is invariant, and if we remove this the remaining matrix is in the 
adjoint representation of $S L(2 \mid 2)$; the invariants under the adjoint action of this group are then given by the Casimirs which are the independent supertraces from second to fourth order in the $N$ 's. In other words, one adds to the list of invariants the supertraces of the $N_{i}$ 's from first to fourth order. It is not difficult to see that these supertraces are fully invariant, and not just quasi-invariant.

The procedure can be repeated for any pair of points $(i, j)$ chosen at the intial step; i.e. by replacing $u_{1}, u_{2}$ by $u_{i}, u_{j}$ and then carrying through the same construction. In this way one generates a large number of quasi-invariants. However, not all of them are independent and it is easy to show that one can replace the invariants of type $k_{i}$ and $m_{3}$ by invariants of the first type. Thus one arrives at the following set of functions: $s_{i j}:=$ sdet $u_{i j}$, for each pair of points, and supertraces of up to the fourth order of the variables of type $N_{i}$, which are defined for each set of four distinct points. The latter are superconformal invariants while the former transform as

$$
s_{i j} \mapsto\left(\operatorname{sdet} h_{i}\right)\left(\operatorname{sdet} h_{j}\right) s_{i j}
$$

One can form full invariants from these by taking cross-ratios, for example of the form

$$
\frac{s_{12} s_{34}}{s_{13} s_{14}}
$$

which is clearly invariant. One thus has two types of invariant: type I, the super cross ratios, and type II, supertraces of products of the $N$ 's up to the fourth power, and of course any function of these. It is not clear how many of these are independent, but we believe that any invariant can be written in terms of them, i.e. they form an overcomplete set.

In order to understand these functions better, it is useful to write them in the standard local coordinate patch. The basic building block, the inverse superdeterminant of $u_{12}$, is the two-point function for an abelian field strength $W$ for which $q=1$; it is

$$
<W(1), W(2)>:=G_{12} \propto s_{12}^{-1}=\frac{y_{12}^{2}}{\hat{x}_{12}^{2}}
$$

where

$$
\hat{x}_{12}=x_{12}-\lambda_{12} y_{12}^{-1} \pi_{12}
$$

and $x_{12}=x_{1}-x_{2}$, etc. Thus the super cross-ratios are simply ratios of ordinary crossratios for the $x$ 's and $y$ 's with supersymmetry being taken care of by the hats. In fact, $\hat{x}_{12}$ and $y_{12}$ are $Q$-supersymmetric variables; one could equally well have chosen to have worked with the $S$-supersymmetric variables, $x_{12}$ and $\hat{y}_{12}=y_{12}-\pi_{12} x^{-1} \lambda_{12}$. The simplest invariant of type II, the supertrace of one $N$ matrix associated with four points $1,2,3$ and 4 , say, can be written in local coordinates as

$$
\operatorname{str} N=\operatorname{str}\left(X_{12}^{-1} X_{23} X_{34}^{-1} X_{41}\right)
$$

Evaluating this, one finds that the leading term is a linear combination of $x$ cross ratios minus the same linear combination of $y$ cross ratios, and the function continues as a power series in $\frac{\lambda \pi}{y}$. 
Higher point functions can be parametrised as free $n$-point functions times invariants, with the external charges taken care of by the free part. As in any conformal field theory, there are invariants from 4 points up, but they are all singular in the $y$ variables. Since the $x$ and $y$ sectors appear symmetrically in the theory, they would also be singular in the $x$ 's if it were not for the fact that these singular points are excluded from the domains of definitions of the functions. However, the internal part of the space really is the entire Grassmannian $\mathbb{G}_{2}(4)$, and so the singular internal points cannot be excluded. On the other hand, it is easy to see that the Green's functions must be perfectly regular in the $y$ 's, and this places very strong constraints on the functions of invariants that can arise.

Since any Green's function transforms as in equation (24), we can write it as the product of two point Green's functions to suitable powers multiplied by a superconformal invariant. The latter can only be a function of the type I and type II invariants we have constructed above. In particular, if we take all the operators in $G$ to have the same charge $N$ for simplicity, the four-point function can be written in the form

$$
G=\left(G_{12}\right)^{N}\left(G_{34}\right)^{N} I
$$

where $I$ is a superconformal invariant function of $X_{1}, \ldots X_{4}$. Therefore the analyticity properties $G$ can be enforced if we know the superconformal invariants. If the function $I$ is composed of only type I invariants, i.e. super cross ratios, then it is easy to convince oneself that analyticity requires that the Green's function be of free field theory form. For example, the free Green's function for four energy-momentum tensors $T$ is of the form

$$
<T(1) T(2) T(3) T(4)>\propto\left(G_{12}\right)^{2}\left(G_{34}\right)^{2}+\text { perms }
$$

One finds that the only choices of invariant functions $I$ consistent with analyticity generate the permuted terms with arbitrary constants.

The situation once we allow for type II invariants is different. Invariants of this type have, in their $(\lambda, \pi)$-expansions singular terms of the form $\left(y_{12}^{2} y_{34}^{2}\right)^{-3}$ and, at first sight, it may appear that that they could not contribute to the above four point Green's function. However, it is possible that such singularities in $y_{i j}$ could be cancelled by zeroes arising from a suitable combination of type I invariants. Although it is clear that analyticity places very strong constraints on the form of the Green's functions, a detailed caculation is required to find if analyticity is sufficiently strong to determine the Green's function up to constants. This calculation is long and complicated, but has been carried out for the four point Green's functions of $\mathrm{N}=2$ analytic operators and will be reported on in detail elsewhere [10]. The result is that such Green's functions can be completely determined for operators of charge two and three, but arbitary functions can occur in the Green's functions of higher charge operators. It is likely that this calculation can be extended to higher point Green's functions in $N=2$ theories with similar conclusions. One would also expect to find that the Green's functions in $N=4$ Yang-Mills theory are determined up to constants by superconformal invariance alone for a class of sufficiently low dimension analytic operators.

We can view the above discussion from a more abstract point of view. According to equation (24) any $N$ point function is an invariant holomorphic section of a line bundle on 
$N$ copies of analytic superspace, the particular line bundle being determined by the $U(1)$ weight of the operators involved. A Green's function will be determined up to constants if there are only a finite number of such sections of the approriate bundle. However, we are unaware of any general theorems that classify the number of such sections.

In some senses it is to be expected that one cannot determine the Green's functions for all operators since this would implicitly require a definition of the theory with an action involving operators of arbitarily high dimension. However, the Green's functions could be further restricted by requiring that they satisfy physical properties such as crossing and unitarity. One may also be able to use the bootstrap programme to determine higher point Green's functions in terms of lower point Green's functions.

Finally, we comment on the heuristic argument given at the end of reference [7]. There it was argued that the form of the operator product expansion for analytic operators involves a finite number of primary fields and one could, as a result, hope to solve for Green's functions in terms of the constants that appear in the operator product expansion. This argument relies on the operator product expansion being meaningful, i.e. convergent. While this may not be the case for operators of high dimension the results given in this paper suggest that it at least seems to be correct for (analytic) operators of sufficiently low dimension. We also note that the argument of reference [7] uses the bootstrap idea which is not used in the arguments presented for solvability in this paper.

It would be of interest to extend the analysis outlined here to spontaneously broken superconformal symmetry. If one could calculate some Green's functions in this phase one might hope to be able to verify the predictions of duality directly. Finally, it is also possible to study anomalous superconformal Ward Identities, for example in $N=2$ theories; this was done in [11] where it was used to derive the 'Matone Identity' [12] for the Seiberg-Witten prepotential 13. This relation was also derived using instanton methods in [15] In an interesting recent paper [14, it has been shown that this identity was sufficent to recover the enitire solution of Seiberg-Witten for the prepotential [13

\section{References}

[1] M. Sohnius and P. West Phys. Lett. B100 (1981) 45; S.Mandelstam, Nucl. Phys. B213 (1983) 149; P.S. Howe, K.S. Stelle and P.K. Townsend, Nucl. Phys. B214 (1983) 519; Nucl. Phys. B236 (1984) 125. L. Brink, O. Lindgren and B. Nilsson, Nucl. Phys. B212 (1983) 401.

[2] P. Howe. K. Stelle and P. West, Phys. Lett 124B (1983) 55.

[3] P. Howe and P. West, "Non-Perturbative Green's Functions in Theories with Extended Superconformal Symmetry", hep-th/9509140, Int. Journal. Mod. Phys. to be published.

[4] A. Galperin, E. Ivanov, S. Kalitzin, V. Ogievetsky and E. Sokatchev, Class. Quant. Grav. 2 (1985) 155, Class. Quant. Grav.1 (1984) 469. 
[5] G. Hartwell and P. Howe, Class. Quant. Grav. 12 (1995) 1823.

[6] H. Osborn, Phys. Lett. B83 (1979) 321, A. Sen, Phys. Lett. 329B (1994) 217.

[7] P. Howe and P. West, Operator Product Expansions in Four-Dimensional Superconformal Field Theories, hep-th/9607060, Phys. LettB to be published.

[8] P. Argyres, M. Plesser, N. Seiberg and E. Witten, Nucl. Phys. B461 (1996) 71.

[9] C. Montonen and D. Olive, Phys. Lett 72B (1977) 117.

[10] P. Howe, A. Pickering, E. Sokatchev and P. West, in preparation.

[11] P.S. Howe and P.C. West, hep-th/9607239, Nucl. PhysB. to appear.

[12] M. Matone, Phys. Lett. B357B (1995) 342.

[13] N. Seiberg and E. Witten, Nucl. Phys. B421 (1994) 484.

[14] G. Bonelli, M. Matone and M. Tonin, hep-th/9610026.

[15] N. Dorey, V. Khoze, and M. Mattis, Phys. Rev. D54 (1996) 2921, F. Fucito and G. Travaglini, hep-th/9605215. 